\title{
Efecto del alga marina Sargassum vulgare C. Agardh en suelo y el desarrollo de plantas de cilantro
}

\author{
Effect of seaweed Sargassum vulgare C. Agardh in soil and the development of \\ coriander plants
}

\author{
María E. Uribe-Orozco ${ }^{1}$, Luz E. Mateo-Cid' ${ }^{1}$ A. Catalina Mendoza-González', Enriqueta Feliciana \\ Amora-Lazcano ${ }^{1}$, Daniel González-Mendoza², Dagoberto Durán-Hernández²*
}

\section{RESUMEN}

En el presente trabajo se evaluó el efecto de la aplicación de harina seca del alga marina (Sargassum vulgare) sobre un suelo pobre y el crecimiento de plantas de cilantro. Los resultados de los análisis realizados al suelo arrojaron que es un suelo arenoso con un $\mathrm{pH}$ de 6.4 y CE de $0 \mathrm{mS} / \mathrm{cm}$. Al finalizar el experimento los tratamientos que modificaron estas variables fueron el de fertilizante con $\mathrm{pH}$ de 5.62 y CE de 1.07 y con $9 \mathrm{~g}$ de harina con $\mathrm{pH}$ de 7.92 y CE de $0.22 \mathrm{mS} / \mathrm{cm}$. Los resultados de la longitud y peso seco total del cilantro indican que desde el punto de visto productivo, los mejores efectos en el desarrollo de la planta son generados por la aplicación de 6 y 9 gramos de harina con una mayor longitud y cantidad de biomasa generada, lo que nos indica que el mayor desarrollo de las plantas de cilantro puede estar influenciada por la materia orgánica y los compuestos bioactivos que aporta la harina al suelo. Futuros estudios deben ser encauzados para determinar el potencial biotecnológico que poseen las algas y sus compuestos bioactivos en aras de un uso agrícola sustentable.

Palabras clave: metales pesados, harina, composición química, conductividad eléctrica.

\begin{abstract}
In the present study our evaluated the effect of the application of seaweed dry meal Sargassum vulgare on poor soil and on the growth of coriander plants was evaluated. The results of the analyzes carried out on the soil showed that it is a sandy soil with a pH of 6.4 and $C E$ of $0 \mathrm{mS} / \mathrm{cm}$, and that at the end of the experiment the treatments that modified these variables were fertilizer with a pH of 5.62 and CE 1.07 and with $9 \mathrm{~g}$ of flour with $\mathrm{pH}$ of 7.92 and CE of $0.22 \mathrm{mS} / \mathrm{cm}$. Results of the total length and dry weight of coriander they indicate that from the productive point of view, the best effects in the development of the plant are generated by the application of 6 and 9 grams of flour with a longer length and amount of biomass generated which indicates that the greater development of coriander plants may be influenced by the organic matter and the bioactive compounds that the flour contributes to the soil. Future studies should be conducted to determine the biotechnological potential of algae and their bioactive compounds for the sake of a sustainable agricultural use.
\end{abstract}

Key words: heavy metals, flour, chemical composition, electric conductivity.

\section{Introducción}

La agricultura a nivel mundial depende de fertilizantes y productos químicos como herbicidas, insecticidas y fungicidas para controlar plagas y enfermedades en los cultivos. Logrando elevar la producción agrícola y a la vez contribuyendo al deterioro ambiental (Pérez y Landeros, 2009). Por lo

\footnotetext{
${ }^{1}$ Escuela Nacional de Ciencias Biológicas, Instituto Politécnico Nacional, Cuidad de México, México.

2 Instituto de Ciencias Agrícolas, Universidad Autónoma de Baja California. Carretera a Delta s/n. Ejido Nuevo León, Mexicali, Baja California, México.

*Autor de correspondencia: ddurn@uabc.edu.mx
}

Fecha de Recepción: 29 mayo, 2018.

Fecha de Aceptación: 24 julio, 2018.

DOI: http://dx.doi.org/10.4067/S0718-34292018005001202. Publicado en línea: 3-diciembre-2018. 
tanto, existe la necesidad de buscar alternativas para la agricultura y que éstas no sean agresivas con el ambiente.

Desde la antigüedad las algas marinas se han utilizado como mejorador del suelo por su alto contenido de fibra y de minerales (Cabioch, 1976), en estado fresco se incorporan a los suelos de cultivo, además se deshidratan las algas para elaborar harina y extractos, usados en los suelos, tratamientos foliares y en invernaderos (Metting et al., 1990), beneficiando los cultivos por su aporte de diversos compuestos y contenido de reguladores de crecimiento (Norrie, 2005). Actualmente, la mayoría de los productos comerciales para la agricultura a base de algas son elaborados a partir de las grandes algas pardas ocupando el primer lugar en términos de importancia económica (Robledo y Freile-Pelegrín, 1998). El género Sargassum es el más abundante y dominante en las zonas rocosas donde forma mantos en aguas tropicales y templadas en el mundo (Critchley y Ohno, 1998). Por lo que consideramos que las algas de este género tienen un gran potencial para su explotación y uso en la agricultura. Por lo antes mencionado, se planteó la siguiente hipótesis de que a mayor cantidad de harina de Sargassum vulgare, se aportaran más nutrientes al suelo, esperando algún cambio en los parámetros físicoquímicos del mismo, que favoreciera el crecimiento de cilantro (Coriandrum sativum var. Fiesta Green). El objetivo de la investigación fue evaluar el efecto de la harina de Sargassum vulgare en el suelo y el crecimiento del cilantro.

\section{Materiales y métodos}

\section{Recolecta del alga y preparación de la harina}

La recolecta de Sargassum vulgare se realizóen la playa el Pulpo, Barra de Cazones, Municipio Cazones de Herrera, Veracruz, ubicada entre las coordenadas $20^{\circ} 43$ ' 33.98" N y $97^{\circ} 11^{\prime} 90.72$ ' W. Las algas se enjuagaron con agua de mar para eliminar arena y organismos adheridos al talo, se dejaron escurrir para su traslado al Laboratorio de Ficología de la Escuela Nacional de Ciencias Biológicas del Instituto Politécnico Nacional (ENCB-IPN).

Para eliminar el exceso de sales, las algas fueron lavadas con agua corriente (cinco veces) y con agua bidestilada (tres veces) y posteriormente fueron colocadas a secado a $60^{\circ} \mathrm{C}$ en una estufa de aireación forzada (Thermo Scientific ${ }^{\mathrm{TM}}$ Precision $^{\mathrm{TM}}$ ), durante 8 días.
Las algas deshidratadas se trozaron manualmente y se trituraron en un molino eléctrico. A la harina obtenida se le realizaron análisis fisicoquímicos en el Laboratorio de Ingeniería Bioquímica (ENCB, IPN), basados en diferentes métodos según las NOM116-SSA1-1994, NMX-F-615-NORMEX-2004, NOM-051-SCFI/S SA 1-2010, NMX-F-608NORMEX-2002, NMX-F-607-NORMEX-2002, NOM-068-SSA1-1994, NOM-051-SCFI/SSA1-2010; se determinaron los metales pesados (Cobre, Cadmio, Cromo, Níquel, Plomo y Zinc), en el Laboratorio de Análisis y Monitoreo Ambiental del Centro Interdisciplinario de Investigaciones y Estudios sobre Medio Ambiente y Desarrollo (CIIEMAD-IPN), a través de la técnica de espectrofotómetro de absorción atómica; la medición de $\mathrm{pH}$ y conductividad eléctrica (CE), en relación agua-suelo de 2:1 por triplicado, se realizó en el Laboratorio de Microbiología del Suelo (ENCB-IPN), con un potenciómetro y conductímetro de tipo HANNA. El resto de la harina se almacenó hasta su posterior uso, en los tratamientos.

\section{Análisis de suelo}

La muestra de suelo se obtuvo en el barrio de Xochiaca, Chimalhuacán, Estado de México, ubicado en las coordenadas $19^{\circ} 44^{\prime} 56$ N y $98^{\circ} 94^{\prime} 22^{\prime \prime}$ W. Se sacó suelo aleatoriamente en el terreno a una profundidad de $20 \mathrm{~cm}$ y se homogenizó en un contenedor (FAO, 2002). Se determinó la textura del suelo con la técnica de Bouyoucus en el Laboratorio de Microbiología del Suelo (ENCB-IPN) y se le determinó el contenido de metales pesados de forma similar al análisis realizado previamente a las algas. (Cobre, Cadmio, Cromo, Níquel, Plomo y Zinc); los análisis de pH y conductividad eléctrica, se realizaron en tres etapas: 1era etapa: al suelo recolectado sin tratar, 2da etapa: a los 35 días posteriormente de haber aplicado la harina de $S v$ y a los 125 días del experimento cuando las plantas tenían 90 días de haberse sembrado, de cada uno de los tratamientos se tomaron tres muestras de $20 \mathrm{~g}$ considerando las réplicas.

\section{Establecimiento del cultivo}

Para medir el efecto de la harina de $S v$ sobre el suelo y el cilantro, se evaluaron los tratamientos siguientes: 1) Testigo, 2) $3 \mathrm{~g}$ de Fertilizante triple 17 (17N-17P-17K), 3) $3 \mathrm{~g}$ de harina de $S v, 4) 6 \mathrm{~g}$ de harina de $S v$ y 5) $9 \mathrm{~g}$ de harina de $S v$, se emplearon 3 repeticiones con 4 vasos de unicel de medio litro 
como unidad experimental por tratamiento. En primera instancia se adicionó la muestra de suelo a cada uno de los vasos de los tratamientos hasta $3 / 4$ de su capacidad. En seguida se agregó la harina de $S v$ solo a los tratamientos con $3 \mathrm{~g}, 6 \mathrm{~g}$ y $9 \mathrm{~g}$, colocándola a una profundidad de $2 \mathrm{~cm}$, homogeneizando y cubriendo con suelo (FAO, 2002), y se dejó actuar por 35 días. Transcurrido el tiempo establecido en los tratamientos se procedió a la siembra, para lo cual se emplearon semillas comerciales de cilantro var. Fiesta Green. Sembrando manualmente 3 semillas a una profundidad no mayor a $1,5 \mathrm{~cm}$ y se rego periódicamente para mantener el suelo húmedo (Morales-Payán et al., 2011). Al mes posterior a la siembra se eliminaron las plantas pequeñas en todos los tratamientos para dejar plantas de tamaño uniforme y se agregaron los $3 \mathrm{~g}$ de fertilizante (triple 17), al tratamiento respectivo.

\section{Variables de crecimiento}

Transcurridos 90 días después de la siembra, se evaluaron las variables de longitud total $(\mathrm{cm})$ y peso seco total (g) de las plantas de cilantro. Se midieron a partir del nodo, longitud foliar y la longitud radicular con un flexómetro, posteriormente se pesaron en una báscula de doble platillo Cent-o-Gram capacidad de $311 \mathrm{~g} \pm 0,5$ gramos y se colocaron en una estufa de secado (Thermo Scientific ${ }^{\mathrm{TM}}$ Precision $^{\mathrm{TM}}$ ) por 8 días, para determinar el peso seco.

\section{Análisis estadístico}

Se llevó a cabo un análisis estadístico utilizando el programa de Diseños Experimentales de la Facultad de Agronomía de la Universidad Autónoma de Nuevo León (FAUANL), versión 2.1 (Olivares, 1990), los datos que se obtuvieron en los tratamientos fueron sometidos a análisis de varianza y mediante la prueba de Tukey $(\mathrm{p}<0,05)$, se hizo la comparación de medias, para todos los casos.

\section{Resultados y discusión}

\section{Análisis de la harina de Sargassum vulgare}

Para conocer el potencial biotecnológico de Sargassum vulgare como posible mejorador de suelo se determinó la composición fisicoquímica de la harina. Los resultados del análisis fisicoquímico realizado sobre $100 \mathrm{~g}$ de materia seca determinaron un contenido de $9.0 \mathrm{~g}$ de proteínas, $1.0 \mathrm{~g}$ de lípidos y $28 \mathrm{~g}$ de fibra cruda (Tabla 1). En este contexto, (Burkholder et al., 1971) reportan para Sargassum platycarpum ( $S p$ ) 6,89 g de proteínas, 0,37 g de lípidos y 7,95 g de fibra, para Sargassum rigidulum ( $S r)$ 5,92 g proteínas, 0,35 g lípidos, 8,20 g fibra y de Sargassum landigerum $(\mathrm{Sl})$ 6,37 g proteínas, $0,45 \mathrm{~g}$ lípidos y 7,94 g fibra. De esta manera podemos verificar que la harina de $S v$ contiene mayor aporte de proteínas, lípidos y fibra que las especies antes mencionadas.

Tabla 1. Análisis fisicoquímico de la harina de Sargassum vulgare.

\begin{tabular}{lc}
\hline Determinación & Resultados en $\mathbf{1 0 0} \mathbf{g}$ \\
\hline Perdida por secado (Humedad) & $11,00 \mathrm{~g}$ \\
Grasas (lípidos) & $1,00 \mathrm{~g}$ \\
Fibra cruda & $28,00 \mathrm{~g}$ \\
Proteína* & $9,0 \mathrm{~g}$ \\
Cenizas & $15,00 \mathrm{~g}$ \\
Carbohidratos totales & $36,00 \mathrm{~g}$ \\
Aporte energético & $189 \mathrm{kcal}, 802 \mathrm{~kJ}$ \\
\hline FFactor 6,25
\end{tabular}

Sin embargo, los carbohidratos totales para las especies mencionadas son 48,70 $\mathrm{g}(\mathrm{Sp}), 44,84 \mathrm{~g}(\mathrm{Sr})$ y $41,55 \mathrm{~g}(S l)$ y cenizas $36,78 \mathrm{~g}$, 40,70 g y $43,70 \mathrm{~g}$, respectivamente. Mientras que la harina de $S v$ aporto solo $36 \mathrm{~g}$ de carbohidratos totales y de cenizas $15 \mathrm{~g}$.

Los compuestos de las algas marinas se modifican a lo largo del año debido a la variación de los nutrientes y condiciones ambientales en donde se desarrollan (Polat y Ozogul, 2008).

$\mathrm{El}$ análisis de metales pesados en la harina de $S v$ arrojó que el Zinc, Plomo, Níquel y Cobre, presentan los niveles más altos con 17,604, 8,002, 6,001, 3,251 $\mathrm{mg} / \mathrm{kg}$ y en menor concentración el Cromo y Cadmio con valores de 1,400,1,025 mg/kg respectivamente (Tabla 2). El Pb, Cr y el Cd, se consideran metales tóxicos y son de los más estudiados. Villares et al. (2002), informan que los contenidos de minerales pesados en el ambiente marino varían constantemente, además, intervienen otros factores como el $\mathrm{pH}$ y salinidad, que modifican el contenido en las algas.

Por lo que consideramos que el rio Cazones contribuye con el aporte de diferentes compuestos, entre ellos metales pesados, ya que éste desemboca cerca de la zona donde se recolectó el alga. El curso del río es alimentado por varios afluentes en los cuales se vierten desechos de las diferentes actividades como 
Tabla 2. Concentración de metales pesados de la harina de Sargassum vulgare.

\begin{tabular}{lc}
\hline Metal & Valores $(\mathbf{m g} / \mathbf{k g})$ \\
\hline $\mathrm{Cu}$ & 3,251 \\
$\mathrm{Cd}$ & 1,025 \\
$\mathrm{Cr}$ & 1,400 \\
$\mathrm{Ni}$ & 6,001 \\
$\mathrm{~Pb}$ & 8,002 \\
$\mathrm{Zn}$ & 17,604 \\
\hline
\end{tabular}

la agricultura, minería, industrias, asentamientos urbanos, que han contribuido a la modificación del medio marino (INE, 1985).

$\mathrm{Al}$ realizar la medición de $\mathrm{pH}$ y $\mathrm{CE}$ de la harina de $S v$ (Tabla 3), se obtuvo un $\mathrm{pH}$ de 6,38, considerado moderadamente ácido y la CE tiene un valor de 12,90 $\mathrm{mS} / \mathrm{cm}$, fuertemente salino de acuerdo a la NOM021-REC-NAT-2000. Con base en estos resultados es posible determinar que la harina tiene una elevada concentración de elementos que contribuyeron a la modificación del $\mathrm{pH}$ y $\mathrm{CE}$ del suelo. El alto nivel de la CE, no afectó el crecimiento de las plantas de cilantro, lo cual podría atribuirse al tipo de suelo, tiene un buen drenaje, sin embargo, en suelos arcillosos mal drenados, las altas concentraciones de sodio, podrían ser un problema (Qadir y Oster, 2004).

Tabla 3. Análisis de $\mathrm{pH}$ y Conductividad eléctrica (CE) de la harina de Sargassum vulgare.

\begin{tabular}{ll}
\hline $\mathbf{p H}$ & $\mathbf{C E}(\mathbf{M s} / \mathbf{c m})$ \\
\hline 6,38 & 12,90 \\
\hline
\end{tabular}

\section{Análisis de metales pesados y análisis físico del suelo}

Los resultados obtenidos del análisis de los metales pesados en el suelo (Tabla 4), los elementos que presentan los valores más altos son Níquel, Zinc y Plomo con 41,184, 30,227 y 12,184 mg/kg y los niveles más bajos son Cromo 10,783, Cobre 5,891 y Cadmio 0,574 mg/kg. De acuerdo, con lo reportado por Bowie y Thornton (1985), los niveles permitidos para estos elementos en suelos agrícolas son Ni 2-100; Zn 25-200; Pb 10-150, Cr y Cd no los reportan y el $\mathrm{Cu}$ 60. Linsay y Norvell (1978) reportan al Cd <1-2 $\mathrm{mg} / \mathrm{kg}$ y Cromo $1-1000 \mathrm{mg} / \mathrm{kg}$, un rango común en los suelos.

Por otra parte, se consultaron normas internacionales (Canadá, Japón, Holanda) y los valores obtenidos de los metales pesados evaluados
Tabla 4. Concentración de metales pesados en el suelo.

\begin{tabular}{ll}
\hline Metal & Valores $(\mathbf{m g} / \mathbf{k g})$ \\
\hline $\mathrm{Cu}$ & 5,891 \\
$\mathrm{Cd}$ & 0,574 \\
$\mathrm{Cr}$ & 10,783 \\
$\mathrm{Ni}$ & 41,184 \\
$\mathrm{~Pb}$ & 12,184 \\
$\mathrm{Zn}$ & 30,227 \\
\hline
\end{tabular}

no superan los valores establecidos por las normas consultadas. Los metales pesados presentes en el suelo sumados con las cantidades de la harina de $S v$, se determinó que estas no exceden los límites permitidos en la agricultura de acuerdo a la norma oficial mexicana NOM-147-SEMARNAT/SSA12004, la cual establece las concentraciones máximas permisibles de metales pesados en los suelos agrícolas.

El análisis físico a la muestra de suelo (Tabla 5), arrojó un contenido de 88,68 \% de arena, 8,008\% de arcilla, y 3,312 \% de limo, estos valores determinaron la clasificación textural, y de acuerdo a los criterios de USDA (2004), es un suelo arenoso que se caracteriza por retener poca agua, se disgrega y tiene un buen drenaje, perdiendo con facilidad los nutrientes (Carrasco y Ortiz, 2011).

Tabla 5. Determinación de la textura del suelo, de acuerdo USDA (2004).

\begin{tabular}{llll}
\hline \% Arena & \% Arcilla & \% Limo & Suelo \\
\hline 88,68 & 8,008 & 3,312 & Arenoso \\
\hline
\end{tabular}

\section{Análisis químico del suelo}

Los análisis químicos realizados al suelo en la primera etapa (Tabla 6) muestran que tiene un $\mathrm{pH}$ de 6,4 y CE de $0 \mathrm{mS} \mathrm{cm}^{-1}$. A los 35 días de adicionar la harina de $S v$, el pH se incrementó a 7,28, 7,29 y 7,51, mientras que la CE aumentó a 0,02, 0,14 y 0,19 , incrementos proporcionales de acuerdo a las concentraciones de harina manejada en los tratamientos de $3 \mathrm{~g}, 6 \mathrm{~g}, 9 \mathrm{~g}$.

La modificación del pH y la $\mathrm{CE}$ en los tratamientos con la harina de $S v$, se atribuye al proceso de descomposición de la harina durante los 35 días trascurridos, periodo durante el cual se liberaron minerales o elementos traza que posee $S$. vulgare. En este sentido, Surey-Gent y Morris, (1987) mencionan que las algas aplicadas al suelo llevan un proceso de descomposición gradual para liberar los 
Tabla 6. Análisis del suelo de pH y Conductividad eléctrica (CE), a los 0, 35 y 125 días.

\begin{tabular}{lllllll}
\hline \multirow{2}{*}{ Tratamientos } & \multicolumn{2}{c}{ Primera etapa } & \multicolumn{2}{c}{ Segunda etapa } & \multicolumn{2}{c}{ Tercera etapa } \\
& $\mathbf{p H}$ & $\mathbf{C E}(\mathrm{mS} / \mathrm{cm})$ & $\mathbf{p H}$ & $\mathbf{C E}(\mathrm{mS} / \mathrm{cm})$ & $\mathbf{p H}$ & $\mathbf{C E}(\mathrm{mS} / \mathrm{cm})$ \\
\hline Testigo & 6,4 & 0 & $6,4 \mathrm{~b}$ & $0 \mathrm{c}$ & $6,52 \mathrm{~b}$ & $0,15 \mathrm{~d}$ \\
3 g Fertilizante & 6,4 & 0 & $6,4 \mathrm{~b}$ & $0 \mathrm{c}$ & $5,62 \mathrm{c}$ & $1,07 \mathrm{a}$ \\
3 g de harina Sv & 6,4 & 0 & $7,28 \mathrm{a}$ & $0,02 \mathrm{c}$ & $6,31 \mathrm{~b}$ & $0,25 \mathrm{~b}$ \\
6 g de harina Sv & 6,4 & 0 & $7,29 \mathrm{a}$ & $0,14 \mathrm{~b}$ & $6,55 \mathrm{~b}$ & $0,19 \mathrm{~cd}$ \\
9 g de harina Sv & 6,4 & 0 & $7,51 \mathrm{a}$ & $0,19 \mathrm{a}$ & $7,92 \mathrm{a}$ & $0,22 \mathrm{bc}$ \\
\multicolumn{2}{l}{ Las letras diferentes presentan diferencias significativas, según la prueba de Tukey $(\mathrm{p}<0,05)}$.
\end{tabular}

nutrientes al mismo. Lo que las hace un recurso con potencial biotecnológico, para la aplicación como abono en suelos dedicados a la agricultura y suelos con problemas de productividad.

Después de 125 días, se realizó las últimas mediciones de pH y CE en el suelo (Tabla 6), el $\mathrm{pH}$ de los tratamientos testigo, el de $3 \mathrm{~g}$ y $6 \mathrm{~g}$ de harina de $S v$, presentaron un valor similar al inicial. Mientras que los tratamientos con $3 \mathrm{~g}$ de fertilízate $\mathrm{y}$ el de $9 \mathrm{~g}$ de harina tuvieron efectos contrarios, en el primer caso, disminuyó el pH a 5,62, considerado como un suelo moderadamente ácido y en el segundo caso, incrementó a 7,92, clasificado como un suelo medianamente alcalino, de acuerdo a NOM-021REC-NAT-2000.

Por otro lado, la CE incrementó en los tratamientos entre $0,15 \mathrm{a} 0,25 \mathrm{mS} / \mathrm{cm}$, permaneciendo como un suelo no salino. Excepto el tratamiento con $3 \mathrm{~g}$ de fertilizante con $1.07 \mathrm{mS} / \mathrm{cm}$, valor muy cercano a un suelo ligeramente salino (NOM-021REC-NAT-2000). De acuerdo con las modificaciones en las dos variables, el fertilizante tiene un efecto ácido en el suelo. Crouch y van Standen (1993), mencionan que el uso excesivo de fertilizantes en los cultivos afecta el suelo y los ecosistemas. Por otro lado, los suelos que presentan problemas de acidez, se les adiciona $\mathrm{CaCO}_{3}$ o $\mathrm{MgCO}_{3}(\mathrm{FAO}, 2002)$, en concentraciones moderadas, por lo que inferimos que la harina de $S v$, contiene $\mathrm{Ca}, \mathrm{Mg}$ y alginatos, los que están contribuyendo a la modificación del $\mathrm{pH}$, el tratamiento con $9 \mathrm{~g}$ de harina mantuvo un $\mathrm{pH}$ alto de 7,92 al final del experimento, posiblemente se debe a una alta concentración de estos compuestos con base en la cantidad de harina.

En este sentido Badar et al. (2015), mencionan que la adición de fertilizantes orgánicos de algas en cantidades adecuadas mejoran la condición del suelo. Además, incrementan el nivel de nutrientes del suelo como N, P, K, y micronutrientes (SureyGent y Morris, 1987), dichos estudios quedan para próximas investigaciones. Durante el transcurso del experimento, el suelo de los tratamientos con harina de $S v$, presentaron una coloración café que se intensificó con una mayor cantidad de harina, en dichos suelos persistió la humedad, mientras, que en los tratamientos sin harina, el suelo se deshidrataba rápido. Respecto a esto, Surey-Gent y Morris (1987) señalan que la harina aporta materia orgánica y en conjunto con los alginatos, retienen agua, lo cual explica la humedad persistente en el suelo.

\section{Variables fisiológicas}

Transcurridos 90 días de la siembra se evaluaron la longitud total (cm) y peso seco total (g), de las plantas de cilantro. En la figura 1 se muestran los resultados obtenidos de la longitud total $(\mathrm{cm})$, en donde se aprecia que el tratamiento testigo y el de fertilizante (3 g), no presentaron diferencias significativas entre sí, mientras que los tratamientos con 3, 6 y $9 \mathrm{~g}$ de harina $(S v)$ presentaron un incremento gradual de la longitud a mayor concentración de harina, siendo la concentración de $9 \mathrm{~g}$ con la que alcanzaron una mayor elongación con 34,6 cm. Morales-Payán et al. (2011) indican que el cilantro se adapta a una gran variedad de tipos de suelo, sin embargo, se obtiene una mayor producción en suelos fértiles con $\mathrm{pH}$ entre 6,5 y 7,5, con retención de humedad y buen drenaje. Por otra parte, Metting et al. (1990), mencionan que el uso de algas en estado fresco o en harina en el suelo, aportan materia orgánica, nutrientes y compuestos bioactivos (auxinas, giberelinas y citoquinonas) que influyen en el crecimiento de las plantas. Por lo que podemos inferir que la adición de harina en el suelo aportó nutrientes y mejoró las características del suelo, permitiendo con esto que las plantas de cilantro tuvieron un mejor desarrollo vegetativo en comparación con los tratamientos control y con fertilizante. En los tratamientos con $6 \mathrm{~g}$ y $9 \mathrm{~g}$ de harina de $S v$ se obtuvo un peso seco de 0,69 y 0,65 g respectivamente, no 


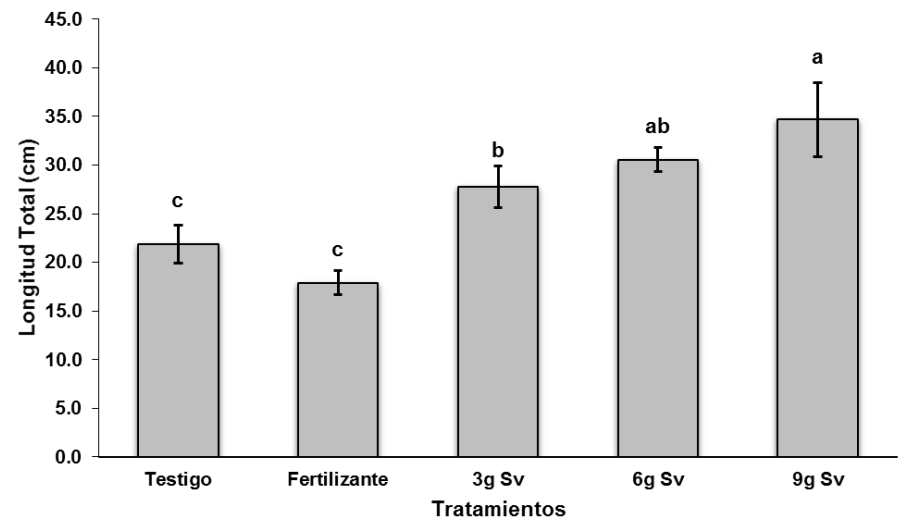

Figura 1. Longitud total $(\mathrm{cm})$ de las plantas de cilantro a los 90 días de la siembra ( \pm std.dev). Tratamientos: T: testigo; F: fertilizante $3 \mathrm{~g}$; harina de Sargassum vulgare (Sv) $3 \mathrm{~g}, 6 \mathrm{~g}$ y 9 g. Las letras diferentes presentan diferencias significativas, según la prueba de Tukey $(\mathrm{p}<0,05)$.

presentando diferencias significativas, mientras que por debajo de los $0,42 \mathrm{~g}$ y no presentan diferencias los tratamientos testigo, fertilizante y $3 \mathrm{~g} S v$ están significativas entre ellos (Figura 2).

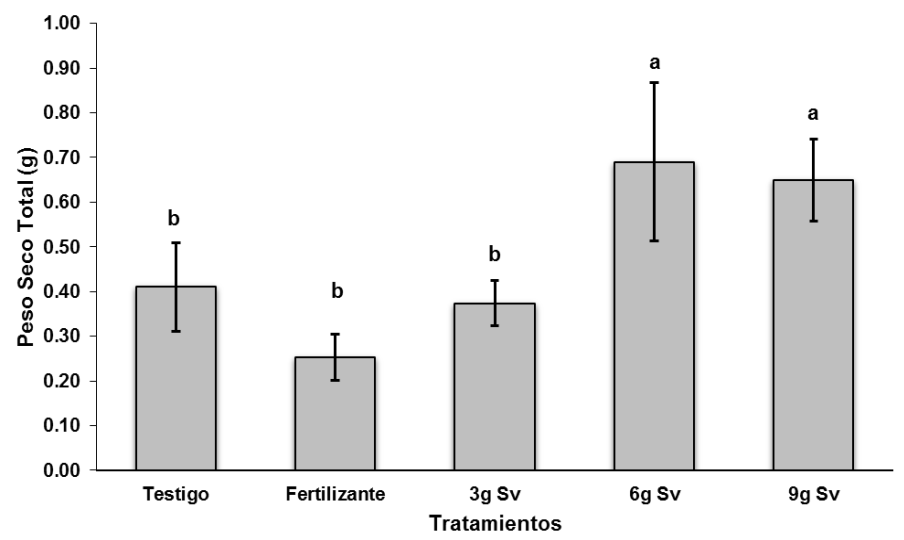

Figura 2. Peso seco total (g) de las plantas de cilantro a los 90 días después de la siembra ( \pm std. dev.). Tratamientos: T: testigo; F: fertilizante $3 \mathrm{~g}$; harina de Sargassum vulgare (Sv) 3 g, 6 g y 9 g. Las letras diferentes presentan diferencias significativas, según la prueba de Tukey $(\mathrm{p}<0,05)$.

Por otro lado, se percibió que en los tratamientos con mayor concentración de harina, la raíz principal disminuía y se incrementaban las raíces adventicias, las cuales presentaban una textura más rígida, mientras que los tratamientos sin harina la raíz era delicada y frágil (Figura 3).
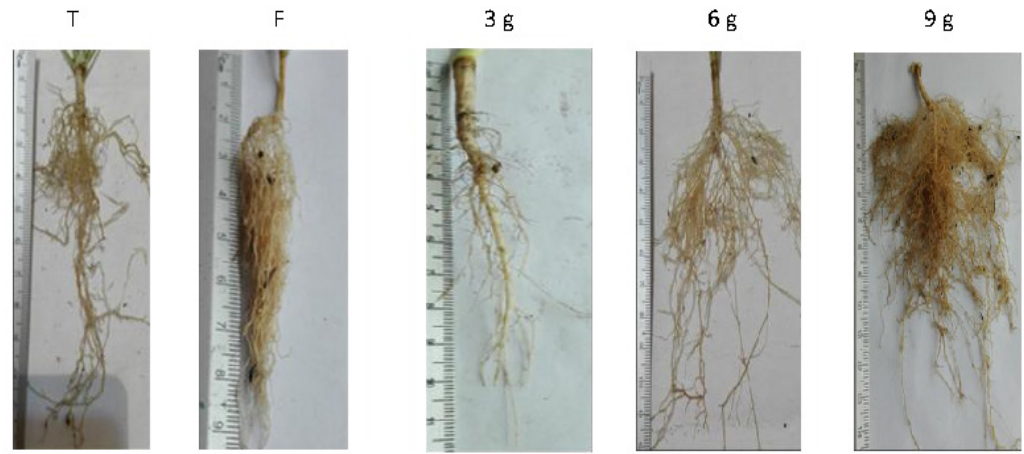

Figura 3. Imágenes de la raíz de cilantro de los cinco tratamientos, a los 90 días de la siembra, T) Testigo; F) $3 \mathrm{~g}$ de fertilizante triple (17N-17P-17K); 3 g, $6 \mathrm{~g}$ y $9 \mathrm{~g}$ de harina de Sargassum vulgare. 
Asimismo, las plantas de cilantro a mayor concentración de harina presentaron más flores y frutos. Estos resultados concuerdan con los mencionados por Metting et al. (1990), quienes indican que las hormonas presentes en la harina de las algas podrían promover el crecimiento de las raíces, además mencionan que los experimentos realizados con diversas plantas, usando productos de algas, tienen un mejor desarrollo de raíz y área foliar, obteniendo así una mayor producción en los cultivos.

De acuerdo a lo anterior, Crouch y van Staden (1992), observaron un aumento de la radícula de las plántulas y la longitud del tallo al aplicar un concentrado de algas al suelo en semillero de tomate. Además, sugieren utilizar concentrados de algas en pequeñas dosis, ya que los compuestos activos deben ser efectivos a bajas concentraciones. Por lo tanto, el tratamiento con 6 y 9 gramos de harina de $S v$ son los que aportaron más biomasa seca, en este tipo de suelo (arenoso). Sin embargo, al no presentar diferencias significativas entre el tratamiento de $6 \mathrm{~g}$ y el de $9 \mathrm{~g}$ de harina de $S v$, podemos considerar el tratamiento con $6 \mathrm{~g}$ el adecuado, debido a que aporto los nutrientes necesarios para el desarrollo de las plantas de cilantro, de esta manera se ahorra el recurso.

\section{Conclusiones}

La harina de Sargassum vulgare tuvo efectos positivos en suelo y en el crecimiento del cilantro, los tratamientos con $6 \mathrm{y} 9 \mathrm{~g}$ de harina son los que aportaron más nutrientes al suelo, observándose cambios en el pH y la CE a partir de los 35 días, lo cual se ve reflejado en las variables evaluadas de longitud total y biomasa seca total del cilantro a los 90 días, por lo tanto, la convierte en un recurso con un gran potencial biotecnológico en la agricultura. Sin embargo; estudios sobre los compuestos bioactivos que posee son necesarios para optimizar y comprender el modo de acción de las algas en la nutrición del suelo.

\section{Agradecimientos}

A la Escuela Nacional de Ciencias Biológicas del Instituto Politécnico Nacional (ENCB-IPN) y al respaldo de los proyectos SIP20161052 Y SIP20164742, por proporcionar las facilidades para llevar a cabo la investigación.

\section{Literatura citada}

Badar, R.; Khan, M.; Batool, B.; Shabbir, S.

2015. Effects of organic amendments in comparison with chemical fertilizer on cowpea growth. Int. J. Appl. Res., 1:66-71.

Bowie, S.; Thornton, I.

1985. Environmental geochemistry and health: report to the Royal Society's British National Committee for Problems of the Environment. D. Reidel Publishing Co. Boston, US. 140p.

Burkholder, P. R.; Burkholder, L. M.; Almodovar, L.R. 1971. Nutritive constitutes of some Caribbean marine algae. Botanica Marina, 14:132-135.

Cabioch, J. 1976. Utilization des Algues. Skol-Vreiz, 45: 20-24.

Carrasco, J.; Ortiz, M.

2011. Propiedades físicas del suelo, que condicionan el desarrollo de frutales en la región de O’Higgins. En: Lemus, G., Salgado, I. (Eds).Centro de Frutales de Carozo: Resumen Técnico. Instituto de Investigaciones Agropecuarias, Rengo, Chile. Boletín INIA 227. Pp. 17-23.

Critchley, A.; Ohno, M, 1998. Seaweed Resources of the World. Japan International Cooperation Agency (JICA), Tokyo, Japan.
Crouch, I.J.; van Staden, J.

1993. Evidence for the presence of plant growth regulators in commercial seaweed products. Plant Growth Regul., 13:21-29.

Crouch, I. J.; van Staden, J.

1992. Effect of seaweed concentrate from Ecklonia-maxima (Osbeck) Papenfuss on Meloidogyne incognita infestation on tomato. J. Appl. Phycol., 5:37-43. FAO.

2002. Los fertilizantes y su uso. Asociación Internacional de la Industria de los Fertilizantes. Paris, Francia. 83p.

INE

1985. Sistema de Información Ecológico a Nivel Paisaje Terrestre para la Cuenca del Rio Cazones. Instituto Nacional de Ecología. México. 215p.

Lahaye, M.

1991. Marine algae as sources of fibers: Determination of soluble and insoluble dietary fiber contents in some sea vegetables. J. Sci. Food Agr., 54: 587-594.

Lindsay, W.L.; Norvell, W.A.

1978.Development of a DTPA soil test for zinc, iron, manganese and copper. Soil Science Society of America Journal, 42:421-428. 
Metting, B.; Zimmerman, W.J.; Crouch, I.;van Staden, J. 1990. Agronomic uses of seaweed and microalgae. In: I. Akatsuka (Ed). Introduction to applied phycology. The Hague: SPB Academic Publishing. pp. 589-627.

Morales-Payán, J.; Bruner, B.; Flores, L.; Martínez, S. 2011. Cilantrillo orgánico, Hoja informativa. Departamento de Cultivos y Ciencias Agroambientales. 8 p.

Norma Oficial Mexicana NOM-021-RECNAT-

2000. Que establece las especificaciones de fertilidad, salinidad y clasificación de suelos. Estudios, muestreo y análisis. Diario Oficial, 31 de diciembre de 2002. Secretaria de Gobierno. México.

Norma Oficial Mexicana NOM-147-SEMARNAT/ SSA1-

2004. Que establece criterios para determinar las concentraciones de remediación de suelos contaminados por arsénico, berilio, cadmio, cromo hexavalente, mercurio, níquel, plomo, selenio, talio y vanadio. Diario Oficial de la Federación, 2 de marzo 2004. Secretaria de Gobierno. México.

Norrie, J.

2005. Aplicaciones prácticas de productos de algas marinas en la agricultura. Tegralia, 15: 26-30.

Olivares, S.E.

1990. Paquete de diseños experimentales FAUANL. Versión 2.1 Facultad de Agronomía, Universidad Autónoma de Nuevo León, (UANL). Marín, N.L. México.

Pérez, A.; Landeros, C.

2009. Agricultura y deterioro ambiental. Elementos: Ciencia y Cultura, 73 (16):19-25.
Polat, S.; Ozogul, Y.

2008. Biochemical composition of some red and brown macroalgae from the northeastern Mediterranean Sea. Int. J. Food Sci. Nutr. 59: 566-572.

Qadir, M.; Oster, J.D.

2004. Crop and irrigation management strategies for salinesodic soils and waters aimed at environmentally sustainable agriculture. Sci Total Environ, 323:1-19.

Robledo, D.; Freile-Pelegrín, Y.

1998. Microflora marina de interés económico de las costas de Yucatán. En: H. Benítez Díaz, E. Vega López, A. Peña Jiménez, S. Ávila Foucat (Eds.). Aspectos económicos sobre la biodiversidad de México. Comisión Nacional para el Conocimiento y Uso de la Biodiversidad/ Instituto Nacional de Ecología (CONABIO-INE) México. pp. 167-179.

USDA

2004. Clasificación textural de los suelos. United State Department of Agriculture.(En línea) Disponible: http://www. miliarium.com/prontuario/Tablas/Suelos/ClasesTexturales. htm. Consultado: 4/sep/2010.

Surey-Gent, S.; Morris, G.

1987. Seaweed: A user's guide. Whitter Books Ltd. London, UK. 160 p.

Villares, R.; Puente X.; Carballeira, A.

2002. Seasonal variation and background levels of heavy metals in two green seaweeds. Environ. Pollut, 119: 79-90. 